\title{
FLORÍSTICA Y DIVERSIDAD DE LAS CIÉNEGAS DEL RÍO LERMA ESTADO DE MÉXICO, MÉXICO
}

\author{
Carmen Zepeda-Gómez ${ }^{1,4}$, Antonio Lot-Helgueras ${ }^{2}$, Xanat Antonio Nemiga ${ }^{3}$ y \\ Delfino Madrigal-UribE ${ }^{3}$ \\ ${ }^{1}$ Universidad Autónoma del Estado de México, Facultad de Ciencias, Instituto \\ Literario 100, 50000 Toluca, Estado de México, México. \\ ${ }^{2}$ Universidad Nacional Autónoma de México, Instituto de Biología, Ciudad \\ Universitaria, 04510 México, D.F., México. \\ ${ }^{3}$ Universidad Autónoma del Estado de México, Facultad de Geografía, Instituto \\ Literario 100, 50000 Toluca, Estado de México, México. \\ 4Autora para la correspondencia: zepedac@uaemex.mx
}

\section{RESUMEN}

Las ciénegas de Lerma o de Chignahuapan, Chimaliapan y Chiconahuapan son áreas naturales protegidas, importantes para la conservación de aves y una de las regiones hidrológicas prioritarias, amenazadas y con alta diversidad biológica en México. No obstante, hay poca información actualizada sobre su vegetación, aun cuando están en franco peligro de desaparecer. En este estudio se identificó la flora vascular acuática y las variaciones de la diversidad vegetal de los tres cuerpos de agua, a partir de la colecta de todas las especies presentes; adicionalmente se aplicó el método de la línea intercepto en 20 a 40 sitios de cada cuerpo de agua. Se encontró una riqueza florística de 97 especies de plantas vasculares incluidas en 39 familias y 65 géneros. De los elementos registrados $46 \%$ fueron acuáticos estrictos, 31\% subacuáticos y 20\% tolerantes. Las familias Cyperaceae, Asteraceae, Lemnaceae, Poaceae, Apiaceae y Polygonaceae fueron las mejor representadas. La diversidad de las ciénegas de Lerma es relativamente alta (índice de Shannon $=3.2$ ) y presenta variaciones particulares en cada humedal. Chiconahuapan muestra los valores más altos y proporciona heterogeneidad florística a los humedales. La prevalencia de malezas terrestres y acuáticas sugiere un deterioro continuo de estos hábitats; sin embargo, la presencia de especies características de áreas lacustres y de distribución restringida indica que las ciénegas aún poseen una diversidad vegetal significativa. Desafortunadamente estos sistemas están sujetos a cambios negativos drásticos e irreversibles, si las condiciones de perturbación, contaminación y disminución del nivel de agua no se revierten. 
Palabras clave: ciénegas de Lerma, Chiconahuapan, Chignahuapan, Chimaliapan, diversidad, florística, México.

\begin{abstract}
The Lerma marshes also known as Chignahuapan, Chimaliapan and Chiconahuapan are natural protected areas and bird conservation zones. They are one of the priority hydrological regions, threatened and highly diverse in Mexico. However there is little updated information about their vegetation, even when they are in clear danger of disappear. This study identified the aquatic vascular flora and the changes in plant diversity of the three bodies of water by means of the collection of all species; additionally we applied the line-intercept method in 20 to 40 sites of each water body. We find 97 species of vascular plants included in 39 families and 65 genera. Forty-six percent are strict aquatic, 31\% subaquatic and 20\% tolerant. The families Cyperaceae, Asteraceae, Lemnaceae, Poaceae, Apiaceae and Polygonaceae are the better represented. The diversity of Lerma marshes is relatively high (Shannon index = 3.2) and presents particular variations in each wetland. Chiconahuapan shows the highest values and provides floristic heterogeneity to wetlands. The prevalence of terrestrial and aquatic weeds suggests a continued deterioration of these habitats; however, the presence of species characteristic of flooded areas and with restricted distribution suggests that the marshes still have significant plant diversity. Unfortunately these systems are subject to drastic and irreversible negative changes, if the conditions of disturbance, pollution and declining water levels are not reversed.
\end{abstract}

Key words: Chiconahuapan, Chignahuapan, Chimaliapan, diversity, floristic, Lerma wetlands.

\title{
INTRODUCCIÓN
}

Las ciénegas pertenecen al grupo de los ecosistemas palustres también conocidos como humedales; se trata de espacios dinámicos que tienen importancia local y regional, ya que regulan el ciclo hidrológico y la calidad del agua (Mitsch y Gosselink, 1993; Tiner, 1999), son hábitats para organismos dulceacuícolas y esenciales para muchas especies terrestres al ser sitios de anidamiento y de residencia de varias aves migratorias (Holland et al., 1990). En un contexto amplio, los humedales son importantes reguladores del flujo de nutrientes y sedimentos entre ambientes 
acuáticos y terrestres (Holland et al., 1990; Mitsch y Gosselink, 1993). Las características particulares de estos cuerpos de agua presuponen variaciones estructurales y funcionales de sus comunidades vegetales, expresadas en las diferentes formas de vida de las hidrófitas (Sculthorpe, 1985). Las plantas acuáticas estrictas y las subacuáticas, además de ser los productores primarios de los cuerpos de agua, juegan un papel esencial en el equilibrio, desarrollo y funcionamiento de los ambientes inundados, ya que los servicios ambientales que proporcionan son fundamentales en la salud, así como en la conservación del hábitat lacustre y en consecuencia en la diversidad biológica (Cronk y Fennessy, 2001; Lot y Novelo, 2004).

Las tres ciénegas del río Lerma son también conocidas bajo el nombre de Chignahuapan, Chimaliapan y Chiconahuapan, forman parte de la cuenca alta del río Lerma, en el centro de México. Estos humedales tienen un papel importante en el almacenamiento e infiltración de agua porque funcionan como vasos reguladores de inundaciones (Pérez-Ortiz y Valdez, 2006), además poseen especies endémicas y nativas en riesgo, algunas de importancia estética y económica (Pérez-Ortiz y Valdez, 2006), por lo que han sido decretadas áreas naturales protegidas (Anónimo, 2002a; Pérez-Ortiz y Valdez, 2006) y de importancia para la conservación de aves (Arizmendi y Márquez, 2000), pues constituyen el hábitat prioritario de numerosos grupos residentes y migratorios (Anónimo, 2006). Además de su riqueza biológica y su funcionalidad ecológica, las ciénegas de Lerma aún son base de actividades productivas y socioculturales. Localmente todavía existen economías extractivas y de sustento basadas en el uso de especies de flora y fauna acuática, pesca artesanal, recolección de invertebrados y plantas, caza tradicional de aves, cultivo en chinampas y pastoreo. Sin embargo, los vestigios de las ciénegas de Lerma se encuentran en un avanzado proceso de contaminación, desecación y fragmentación que ha impactado dramáticamente en la pérdida de su biodiversidad y en las actividades humanas relacionadas con las mismas, como también ocurre en otros cuerpos de agua del centro de México (Lot y Zepeda, 2009).

Toda la cuenca alta de Lerma posee $11.8 \%$ de especies de plantas acuáticas de México, $17 \%$ de los géneros y 36\% de las familias (Ramos, 2000), además de ser la región más rica en familias de hidrófitas estrictas dentro del estado (Lot et al., 1986, Lot et al., 1998). No obstante, existen pocos estudios botánicos de esta región, algunos de ellos sólo citan especies de zonas inundadas dentro del análisis que hacen para grupos de plantas terrestres de toda la entidad mexiquense, como los realizados para gramíneas (Dávila, 2009), orquídeas (Aguirre-León, 2009) y helechos (Tejero-Díez y Arreguín-Sánchez, 2004; Tejero-Díez, 2009). La contribución de Rioja y Herrera (1951) es la primera aportación al conocimiento de la flora del 
valle de Toluca y en particular de la región de Lerma. En el mismo contexto está el trabajo de Ramírez y Herrera (1954). El estudio de Ramos (2000) se considera la investigación más importante sobre la flora acuática de la región, ya que examina la composición florística de ocho tipos de cuerpos de agua artificiales y naturales presentes en toda cuenca alta de Lerma y describe su vegetación en función de las formas de vida de las plantas que crecen en ellos; reporta 277 especies de hidrófitas estrictas, subacuáticas y tolerantes, y concluye que los ambientes lóticos y lénticos de la cuenca se encuentran en un estado de deterioro importante.

Dada la situación que presentan estos humedales y la amenaza sobre su diversidad biológica, surge la necesidad apremiante de conocer la flora que aún albergan. Por ello, el presente trabajo se propone describir la vegetación acuática y la riqueza vegetal en los tres cuerpos de agua que conforman a las ciénegas de Lerma. La información generada puede aportar criterios y establecer las bases para que en el futuro se planeen estrategias de preservación, manejo y uso de los recursos de estos ambientes.

Área de estudio

Este trabajo se realizó en las tres ciénegas de Lerma; 1) Chignahuapan o Laguna de Almoloya del Río, 2) Chimaliapan o Laguna Lerma y 3) Chiconahuapan o Laguna de Atarasquillo. Las áreas pantanosas forman parte del sistema hidrológico Lerma-Chapala-Santiago y se ubican en el curso alto de la cuenca alta del río Lerma en el Estado de México; son los reductos de 27,000 ha de humedales que había en la zona y que formaban un continuo a finales del siglo XIX (Martínez, 1993), actualmente sólo cubren un total de 3023 ha.

La ciénega de Chignahuapan se ubica entre las coordenadas $19^{\circ} 08^{\prime}-19^{\circ} 09^{\prime}$ latitud norte y $99^{\circ} 29^{\prime}$ - 99 $31^{\circ}$ longitud oeste, a una altitud de $2580 \mathrm{~m}$, posee una extensión aproximada de 596 ha. La de Chimaliapan es la más grande de las tres,

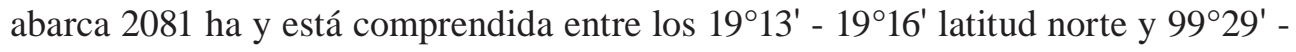
99³1' longitud oeste, a $2560 \mathrm{~m}$ s.n.m. Chiconahuapan se localiza a $2560 \mathrm{~m}$ s.n.m. tiene una extensión de 346 ha y se encuentra entre los $19^{\circ} 20^{\prime}$ - $19^{\circ} 21^{\prime}$ latitud norte y $99^{\circ} 29^{\prime}$ - 99 $31^{\prime}$ longitud oeste (Fig. 1).

La región se caracteriza por el clima templado subhúmedo con lluvias en verano $\left(\mathrm{C}\left(\mathrm{w}_{2}\right)(\mathrm{w}) \mathrm{b}(\mathrm{i}) \mathrm{g}\right)$, con temperatura media anual de $12{ }^{\circ} \mathrm{C}$ y precipitación media anual de 800 a $1200 \mathrm{~mm}$ (Vásquez, 1999). Los suelos predominantes son histosoles, poseen una capa orgánica de $40 \mathrm{~cm}$ o más de profundidad formada por la acumulación de grandes cantidades de material vegetal en áreas inundadas. En las zonas aledañas es posible encontrar cambisoles, andosoles, vertisoles y regosoles. La 
Zepeda-Gómez, et al.: Florística y diversidad de las ciénegas del río Lerma, México

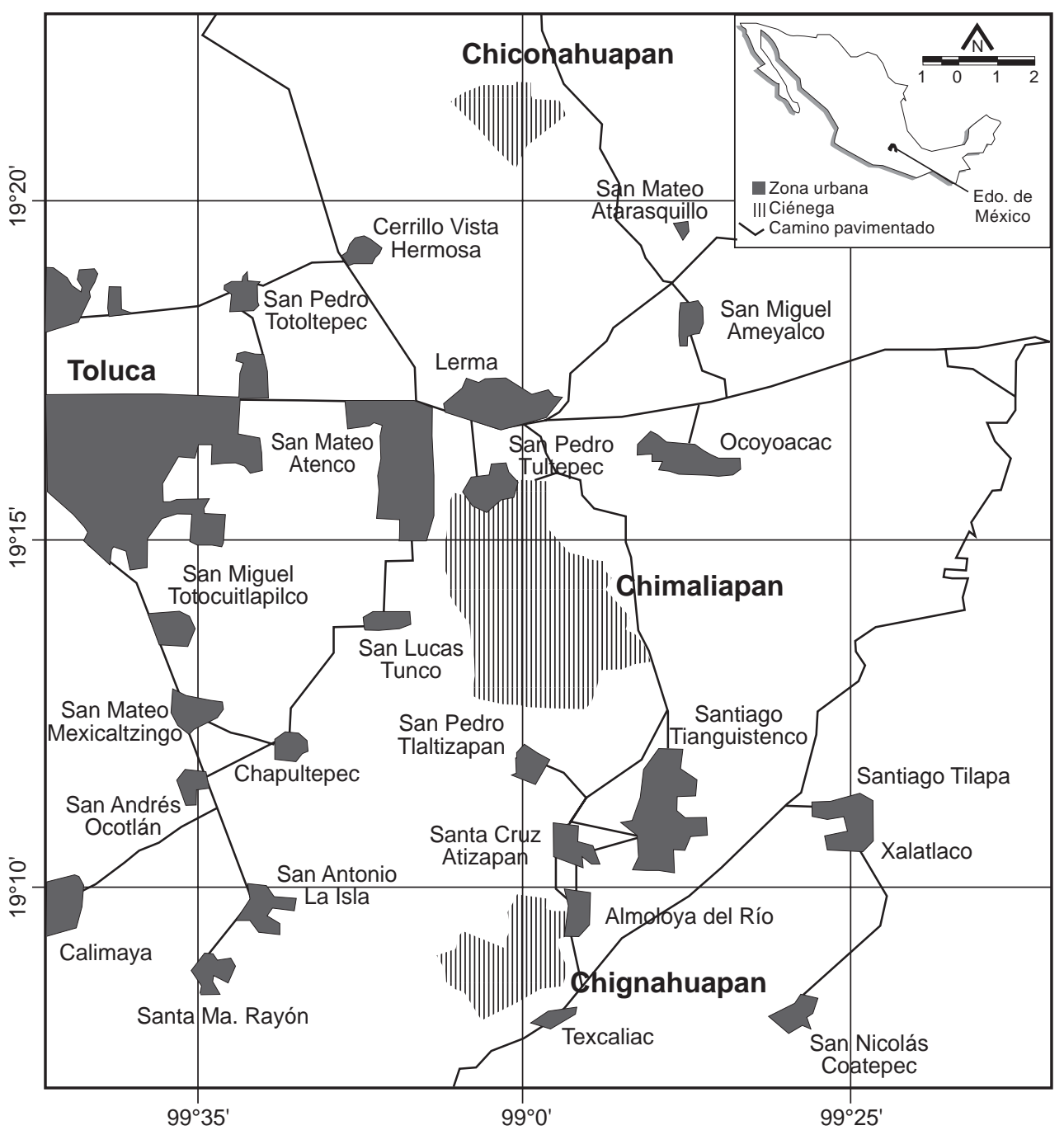

Fig. 1. Ubicación de las tres ciénegas de Lerma en el Estado de México.

composición geológica de la región se encuentra conformada fundamentalmente por rocas clásticas y volcanoclásticas, los depósitos más abundantes son los aluviones (Vásquez, 1999).

En el complejo de humedales de Lerma existen zonas de agua abierta y permanente con una profundidad máxima de $2.5 \mathrm{~m}$, donde prosperan hidrófitas libres 
sumergidas, de hojas flotantes y libres flotadoras, su extensión es menor de $35 \%$ de la superficie actual de cada ciénega. Rodeando estos espacios se extienden amplias franjas sometidas a inundación estacional o ciénegas, en las cuales la profundidad máxima no supera los $120 \mathrm{~cm}$ y donde la vegetación está constituida principalmente por hidrófitas enraizadas emergentes que forman comunidades localmente denominadas tulares (Ramos, 2000). La extensión de los terrenos lacustres en cada ciénega se ve afectada por el régimen de lluvias, durante el año se presentan periodos de inundación de 6 a 8 meses, después de los cuales el nivel del agua disminuye gradualmente hasta crear, en algunos casos, zonas con suelos sólo saturados de humedad. Las variaciones en el nivel de agua de las tres ciénegas han permitido el desarrollo de plantas terrestres tolerantes, las cuales pueden soportar, por cortos periodos de tiempo, suelos inundados o con alta humedad (Ramos, 2000).

\section{MÉTODO}

Flora

En cada ciénega se colectaron, entre mayo y noviembre del 2008, los representantes de todas las plantas acuáticas, subacuáticas y tolerantes presentes y con esta información se elaboró una lista general de especies, la cual se ordenó siguiendo la clasificación de Cronquist (1981) y Brummitt (1992) para las plantas con flor y de Tryon y Tryon (1982) para los helechos. Los nombres de los autores de las especies se abreviaron de acuerdo con Brummitt y Powell (1992). En el caso de las consideradas en riesgo se anotó la categoría de protección según la NOM059-ECOL-2001 (Anónimo, 2002b) y se indicaron las endémicas a Megaméxico de acuerdo con el criterio de Rzedowski (1998). Las clases de plantas acuáticas, subacuáticas, tolerantes y riparias se reportan según Lot y Novelo (1978), Lot et al. (1999) y Rzedowski y Rzedowski (2001), las formas de vida con base en Sculthorpe (1985) y las malezas de acuerdo con Espinosa y Sarukhán (1997) y Rzedowski y Rzedowski (2001).

\section{Clasificación de la vegetación}

Para describir y caracterizar la vegetación dominante de las ciénegas se seleccionaron sistemáticamente de 20 a 40 sitios de muestreo por ciénega. La selección se hizo en función de la magnitud del área inundada y la accesibilidad del terreno. En cada sitio se realizó un levantamiento por el método de línea intercepto de acuerdo con lo propuesto por Matteucci y Colma (1982). Esta técnica es usada en estudios 
de comunidades donde las plantas individuales son difíciles de distinguir y contar (por ejemplo, gramíneas y ciperáceas); en tales casos las evaluaciones y los cálculos respectivos no consideran tallos separados sino grupos de plantas (Brower et al., 1997). Por el tamaño y el agrupamiento de los organismos se utilizaron dos líneas de $10 \mathrm{~m}$ de longitud por sitio de muestreo y en cada una se determinó la presencia de las especies a intervalos de $0.5 \mathrm{~m}$ y se midió la longitud que cada planta o grupo de ellas interceptó en la línea.

Para conocer las especies con mayor valor de importancia (VI) de las ciénegas, se calculó de acuerdo con Brower et al. (1997): 1) la densidad relativa ( $\mathrm{Dr}=$ número de individuos de la especie i/número total de individuos de todas las especies), 2) la cobertura relativa $(\mathrm{Cr}=$ suma de longitudes interceptadas por la especie $\mathrm{i} / \mathrm{suma}$ de longitudes interceptadas de todas las especies), 3) la frecuencia ( $\mathrm{F}=$ número de intervalos interceptados por la especie i/número total de intervalos de la línea), 4) la frecuencia relativa $(\mathrm{Fr}=$ frecuencia de la especie $i /$ suma de frecuencias de todas las especies) y 5) el valor de importancia (VI = suma de los valores relativos de densidad, cobertura y frecuencia).

La presencia de grupos florísticos en las ciénegas en conjunto y para cada una de ellas se determinó mediante un análisis de clasificación con el método de Ward (varianza mínima) con medidas de distancia euclidiana en el programa Statistica 7. Este método permite distinguir conjuntos según su importancia proporcional calculada en función de los valores relativos de densidad, cobertura y frecuencia de cada especie.

\section{Diversidad}

Las estimaciones de diversidad alfa (intra-hábitat) se calcularon usando el índice de Shannon (H’) (Zar, 1999) en el programa Past 1.90 (Hammer et al., 2001). Los valores así obtenidos para cada ciénega se compararon por medio de una prueba de t-Student modificada (Zar, 1999) para establecer diferencias significativas. También se calculó el valor de uniformidad de Pielou $\left(\mathrm{J}^{\prime}\right)$ y el índice de Fisher $(\alpha)$ (Moreno, 2001). La diversidad beta se estimó por el inverso del índice de similitud de Morisita-Horn, calculado mediante un análisis de clasificación utilizando la estrategia aglomerativa de la media aritmética sin ponderación (UPGMA: Unweighted Pair Group Method using Arithmetic averages) para datos de abundancia en el programa Past 1.90 (Hammer et al., 2001). A diferencia de otros métodos, en los cuales sólo se considera la presencia-ausencia de las especies, el cálculo de la similitud de Morisita-Horn permite una comparación cuantitativa de las comunidades, porque incluye valores de riqueza-abundancia de las mismas (Moreno, 2001). El valor obte- 
nido puede variar desde 0 (entidades disímiles) hasta un máximo próximo a uno que indica comunidades idénticas (Brower et al., 1997).

\section{RESULTADOS}

Flora

En las tres ciénegas del Lerma se registraron 95 especies de angiospermas y dos de pteridofitas. Todo el conjunto se agrupa en 39 familias y 65 géneros (Apéndice 1). Las familias mejor representadas fueron: Cyperaceae y Asteraceae con 11 especies, Lemnaceae y Poaceae con 8 y Apiaceae y Polygonaceae con 6, las restantes tuvieron números menores.

De la lista florística, 40 especies (41.2\%) corresponden a plantas acuáticas, 33 (34\%) subacuáticas y 23 (23.7\%) tolerantes (Apéndice 1). Considerando solo las categorías de acuáticas y subacuáticas, el mayor porcentaje (65.7\%) estuvo representado por hidrófitas enraizadas emergentes, seguido de las libre flotadoras $(15 \%)$ y enraizadas sumergidas (6.8\%), mientras que las enraizadas de hojas flotantes, las de tallos postrados y las libre sumergidas tuvieron cada una porcentajes inferiores a 6\%.

Se registraron 10 especies endémicas de México (Apéndice 1) y dos más de Megaméxico. Sobresalen Nymphaea gracilis y Sagittaria macrophylla por estar incluidas como amenazadas en la NOM-059-ECOL-2001, así como Lemna trisulca que se encuentra en la categoría de protección especial.

Clasificación de la vegetación

En el dendrograma que involucra las especies de las tres ciénegas se distinguen cuatro grandes grupos a un nivel de distancia euclidiana de 20 (Fig. 2). El primero se encuentra conformado por Hydrocotyle ranunculoides, Jaegeria bellidiflora, Sagittaria macrophylla, Eleocharis macrostachya y Schoenoplectus californicus, que presentaron los valores de importancia más altos. El segundo conjunto incluye a Paspalum distichum, Echinochloa holciformis, Polygonum punctatum, Lilaeopsis schaffneriana, Eleocharis densa, Myriophyllum heterophyllum, Poa annua y Ranunculus cymbalaria. En los grupos tercero y cuarto se incluyen especies con valores de importancia inferiores a 0.09, en el tercero Berula erecta fue la planta con el valor de importancia más alto, mientras que en el cuarto fue Nymphoides fallax (Apéndice 2 y Fig. 2).

Cada humedal presenta asociaciones particulares. En la ciénega Chignahuapan se registraron 22 especies, de las que el mayor porcentaje correspondió a subacuáticas 


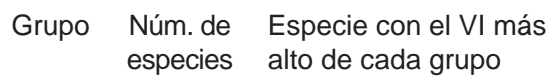

\begin{tabular}{|c|c|c|}
\hline \multicolumn{2}{|c|}{ Chignahuapan } & \multirow[b]{2}{*}{ Eleocharis macrostach } \\
\hline I & 7 & \\
\hline II & 4 & Hydrocotyle verticillata \\
\hline III & 11 & Epilobium ciliatum \\
\hline \multicolumn{3}{|c|}{ Chimaliapan } \\
\hline I & 3 & Sagittaria macrophylla \\
\hline II & 5 & Leersia hexandra \\
\hline III & 6 & Paspalum distichum \\
\hline IV & 13 & Glyceria fluitans \\
\hline
\end{tabular}

\section{Chiconahuapan}

$\begin{array}{lcll}\text { I } & 1 & \text { Myriophyllum heterophyllum } \\ \text { II } & 5 & \text { Typha latifolia } \\ \text { III } & 6 & \text { Hydromystria laevigata } \\ \text { IV } & 13 & \text { Epilobium ciliatum }\end{array}$

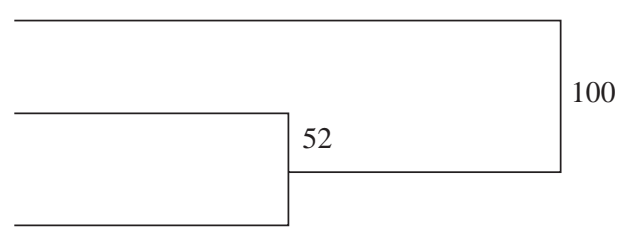

\section{Chignahuapan, Chimaliapan y Chiconahuapan}

$\begin{array}{lcll}\text { I } & 5 & \text { Hydrocotyle ranuncul } \\ \text { II } & 8 & \text { Paspalum distichum } \\ \text { III } & 11 & \text { Berula erecta } \\ \text { IV } & 24 & \text { Nymphoides fallax }\end{array}$

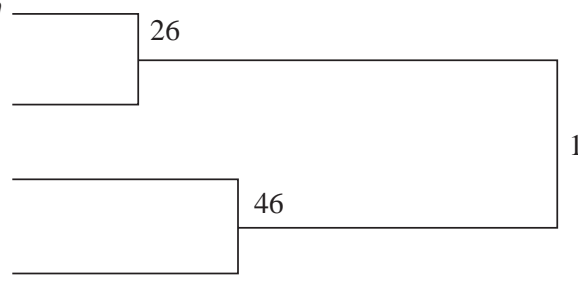

Fig. 2. Diagramas de clasificación de la vegetación de las ciénegas de Chignahuapan, Chimaliapan y Chiconahuapan. Método de Ward con distancias euclidianas. Para cada ciénega se indica en número romano los grupos florísticos, el número de especies que componen a cada grupo y la especie con valor de importancia (VI) más alto en cada grupo. Los números arábigos indican la distancia en porcentaje a la que se fusionan los grupos. 
(55\%), de las tres formas de vida que se presentaron en este humedal, las hidrófitas enraizadas emergentes fueron las más abundantes con $77 \%$ (Fig. 3). La clasificación permitió distinguir tres grupos (Fig. 2). El primero estuvo constituido por siete elementos: Eleocharis macrostachya, Schoenoplectus californicus, Poa annua, Ranunculus cymbalaria, Hydrocotyle ranunculoides, Lilaeopsis schaffneriana y Echinochloa holciformis, que resultaron ser las plantas con los valores de importancia más altos y que definieron a la comunidad vegetal de la ciénega. El segundo se caracterizó por la presencia de Hydrocotyle verticillata, Polygonum mexicanum, Jaegeria bellidiflora y Polygonum punctatum. El tercero estuvo conformado por 11 especies con los valores de importancia más bajos, Epilobium ciliatum, Aster subulatus, Rorippa pinnata, Arenaria paludicola, Eleocharis densa, Polygonum lapathifolium, Rumex crispus, Arenaria bourgaei, Urtica dioica, Mimulus glabratus y Lilaea scilloides.

En la ciénega de Chimaliapan se registraron 27 especies, de las cuales 52\% correspondió a plantas acuáticas y subacuáticas, de éstas $67 \%$ fueron hidrófitas enraizadas emergentes (Fig. 3). La clasificación de las muestras de vegetación generó cuatro grupos (Fig. 2). Los tres elementos del conjunto I (Sagittaria macrophylla, Jaegeria bellidiflora e Hydrocotyle ranunculoides) presentaron los valores de importancia más altos. El grupo II se caracterizó por Leersia hexandra, Eichhornia crassipes, Myriophyllum aquaticum, Lilaeopsis schaffneriana y Polygonum lapathifolium. El III estuvo conformado por Paspalum distichum, Eleocharis macrostachya, Eleocharis densa, Echinochloa holciformis, Berula erecta y Polygonum punctatum. Los organismos con los valores de importancia más bajos se concentraron en el grupo IV y fueron Glyceria fluitans, Aster subulatus, Eleocharis montana, Ludwigia peploides, Eleocharis bonariensis, Lilaea scilloides, Juncus effusus, Polygonum mexicanum, Hydromystria laevigata, Potamogeton nodosus, Schoenoplectus tabernaemontani, Polygonum hydropiperoides y Nymphaea gracilis.

La ciénega de Chiconahuapan presentó el mayor número de especies registradas (29), siete formas de vida y sólo 3\% de tolerantes (Fig. 3). Al clasificar las muestras de vegetación destacan cuatro grupos florísticos (Fig. 2). El conjunto I se conformó únicamente por Myriophyllum heterophyllum como resultado de su alto valor de importancia. El segundo estuvo formado por Typha latifolia, Schoenoplectus californicus, Paspalum distichum, Hydrocotyle ranunculoides y Ludwigia peploides, que junto con Myriophyllum heterophyllum definieron a la vegetación de esta ciénega. Hydromystria laevigata, Jaegeria bellidiflora, Nymphoides fallax, Myriophyllum aquaticum, Eleocharis densa, Potamogeton illinoensis, Lilaeopsis schaffneriana, Polygonum punctatum, Polygonum hydropiperoides y Potamogeton nodosus constituyeron la asociación III. Trece especies con los menores valores de 
Chiconahuapan

Chimaliapan

Chignahuapan

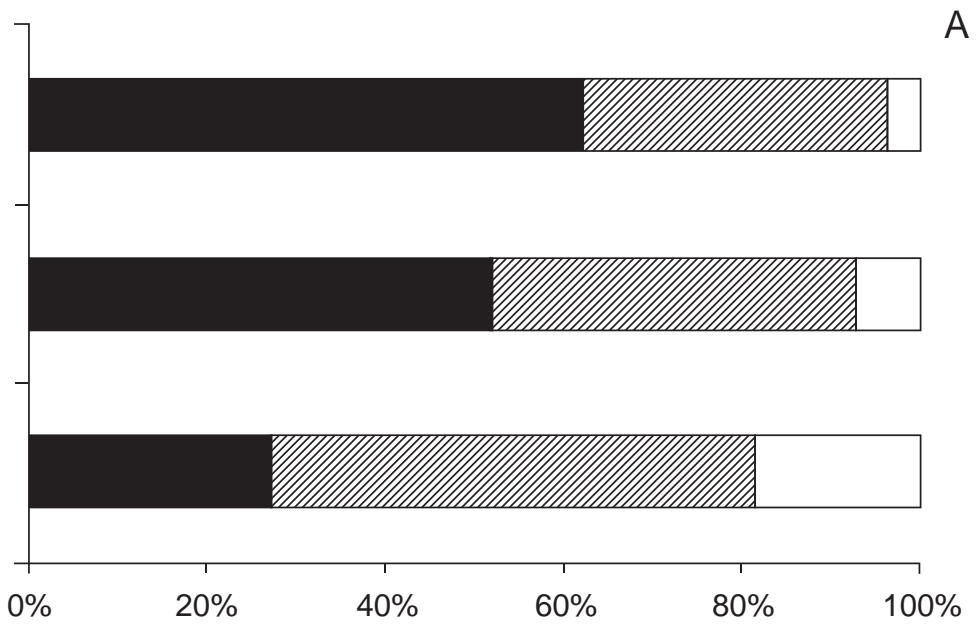

Acuáticas

Qubacuáticas

$\square$ Tolerantes

Chiconahuapan

Chimaliapan

Chignahuapan

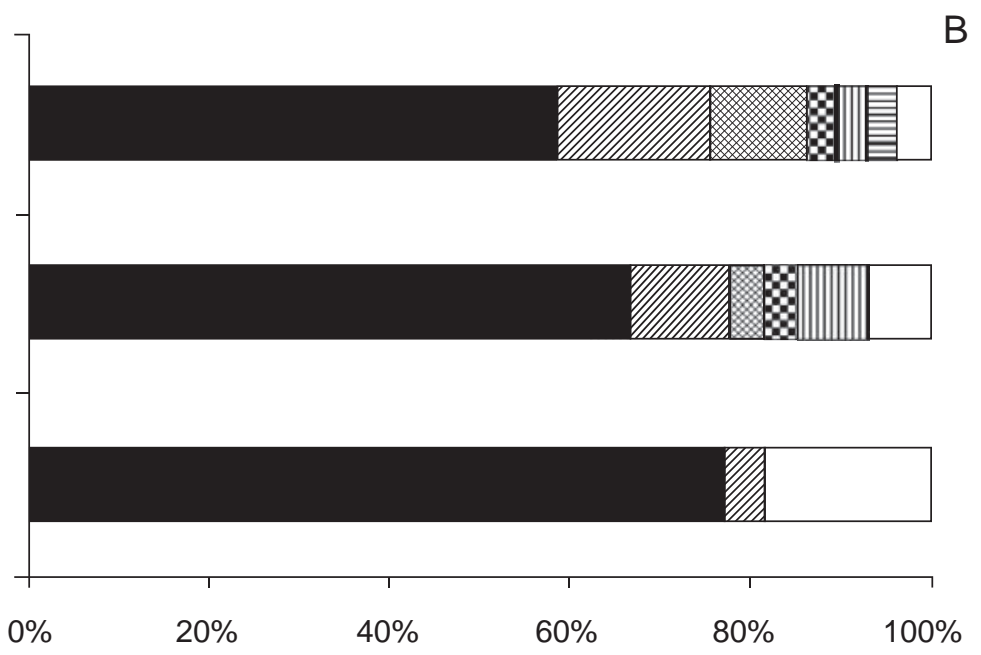

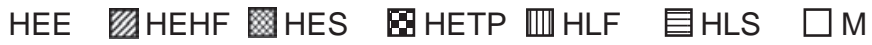

Fig. 3. A) Porcentaje de especies acuáticas, subacuáticas y tolerantes. B) Porcentaje de formas de vida de las plantas de Chignahuapan, Chimaliapan y Chiconahuapan. HEE = hidrófita enraizada emergente, $\mathrm{HEHF}$ = hidrófita enraizada de hojas flotantes, $\mathrm{HES}=$ hidrófita enraizada sumergida, HETP = hidrófita enraizada de tallos postrados, HLS = hidrófita libre sumergida, $\mathrm{HLF}=$ hidrófita libre flotadora y $\mathrm{M}=$ maleza. 
importancias formaron el último agrupamiento de la vegetación acuática de Chiconahuapan: Epilobium ciliatum, Cyperus semiochraceus, Urtica dioica, Berula erecta, Bidens aurea, Eleocharis macrostachya, Schoenoplectus tabernaemontani, Juncus effusus, Eleocharis bonariensis, Jaegeria bellidiflora, Marsilea mollis, Ceratophyllum demersum y Sagittaria macrophylla.

\section{Diversidad}

De acuerdo con el índice de Shannon, la diversidad de toda la comunidad vegetal de las ciénegas de Lerma tuvo un valor general de 3.2. No obstante, analizando cada humedal por separado, los números varían notablemente. Las comparaciones entre pares de ciénegas mostraron diferencias significativas, las cuales se hicieron más evidentes con el cálculo del $\alpha$ de Fisher. La diversidad más alta se presentó en Chiconahuapan que además fue la ciénega más rica (29 especies) y con mayor equidad $\left(\mathrm{J}^{\prime}\right)$. En segundo lugar se ubicó Chimaliapan, sin embargo la estimación de su uniformidad vegetal es inferior a los de las otras dos. Chignahuapan mostró la diversidad más baja y aunque no fue significativamente diferente de la de Chimaliapan, presentó un valor de Pielou ligeramente superior (Cuadro 1).

Cuadro 1. Riqueza de especies, índice de diversidad de Shannon ( $\left.\mathrm{H}^{\prime}\right)$, valor máximo esperado del índice de Shannon (H'max), valor de equidad de Pielou e índice ${ }^{\alpha}$ Fisher de la vegetación de Chignahuapan, Chimaliapan y Chiconahuapan, Estado de México. Las letras diferentes indican diferencias significativas entre pares ciénegas basadas en una prueba de t-Student modificada (Zar, 1999) y con una significancia de 0.05 .

\begin{tabular}{lcccc}
\hline & Chignahuapan & Chimaliapan & Chiconahuapan & Agrupadas \\
\hline Riqueza & 22 & 27 & 29 & 48 \\
Shannon $\left(\mathrm{H}^{\prime}\right)$ & $2.65^{\mathrm{ab}}$ & $2.74^{\mathrm{b}}$ & $2.90^{\mathrm{c}}$ & 3.2 \\
Valor máximo del & 3.09 & 3.29 & 3.36 & 3.8 \\
índice de Shannon & & & & \\
$\left(\mathrm{H}^{\prime}\right.$ max) & & & & \\
Equidad de Pielou $\left(\mathrm{J}^{\prime}\right)$ & 0.858 & 0.833 & 0.864 & 0.855 \\
Índice $^{\alpha}$ de Fisher & 5.0 & 5.1 & 7.3 & 9.1 \\
\hline
\end{tabular}

En general la diversidad alfa calculada para los tres humedales es intermedia tomando en cuenta el valor máximo esperado $\left(\mathrm{H}_{\max }^{\prime}\right.$; Cuadro 1) y el número de especies registradas para cada uno. 
Algunas plantas sólo se detectaron en una ciénega, mientras que otras fueron comunes para las tres zonas, como Eleocharis densa, E. macrostachya, Jaegeria bellidifora, Lilaeopsis schaffneriana, Hydrocotyle ranunculoides y Polygonum punctatum. Usando el Índice de Morisita-Horn se observa que los valores de similitud obtenidos entre pares de zonas en general son bajos, por lo que la diversidad beta es alta (Cuadro 2, Fig. 4). Las zonas estacionalmente inundadas de Chignahuapan y Chimaliapan comparten más especies entre ellas que con Chiconahuapan (Cuadro 2). Chiconahuapan presentó la mayor disimilitud (diversidad beta) cuando se comparó con Chignahuapan y Chimaliapan.

Cuadro 2. Valores del índice de Morisita-Horn (similitud) y diversidad beta (disimilitud en negritas) para pares de ciénegas.

\begin{tabular}{lccc}
\hline & Chimaliapan & Chignahuapan & Chiconahuapan \\
\hline Chimaliapan & 0.00 & $\mathbf{0 . 6 1}$ & $\mathbf{0 . 7 7}$ \\
Chignahuapan & 0.39 & 0.00 & $\mathbf{0 . 7 0}$ \\
Chiconahuapan & 0.23 & 0.30 & 0.00 \\
\hline
\end{tabular}

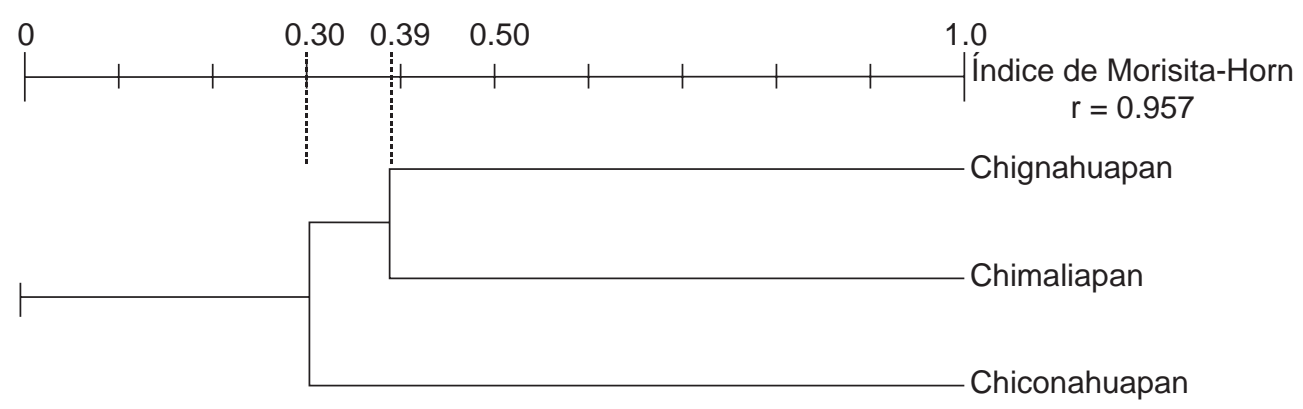

Fig. 4. Diagrama de clasificación de la vegetación de las tres ciénegas de Lerma. Método UPGMA, índice de Morisita-Horn.

\section{DISCUSIÓN}

La riqueza florística de las ciénegas de Lerma es alta a pesar de las pequeñas dimensiones del área que actualmente ocupan $\left(30 \mathrm{~km}^{2}\right)$; sólo es superada 
por la registrada para Tamaulipas, Morelos y Querétaro, sin embargo se trata de inventarios para entidades federativas completas, donde tanto la superficie como la variedad de ambientes acuáticos que albergan son significativamente superiores (Cuadro 3).

Cuadro 3. Número total de especies y de acuáticas estrictas reportadas para diferentes cuerpos de agua de México.

\begin{tabular}{lrcl}
\hline Localidad & $\begin{array}{c}\text { Extensión } \\
\left(\mathrm{km}^{2}\right)\end{array}$ & $\begin{array}{c}\text { Especies } \\
\text { reportadas }\end{array}$ & \multicolumn{1}{c}{ Fuente } \\
\hline Tecocomulco & 17.6 & $36 / 10$ & Lot y Novelo (1978) \\
Yuriria & 97 & $45 / 17$ & Ramos y Novelo (1993) \\
Xochimilco & 26.5 & $48 / 17$ & Novelo y Gallegos (1988) \\
Pátzcuaro & 100 & $48 / 22$ & Lot y Novelo (1988) \\
Zempoala & 47.9 & $66 / 25$ & Bonilla-Barbosa y Novelo (1995) \\
Texcoco & 17 & $78 / 22$ & Rzedowski (1957) \\
Cuitzeo & 3700 & $92 / 25$ & Rojas y Novelo (1995) \\
Zirahuén & 10.48 & $93 / 24$ & Madrigal-Guridi et al. (2004) \\
Tamaulipas & 78380 & $93 / 93$ & Mora-Olivo et al. (2008) \\
Querétaro & 11270 & $118 / ?$ & Martínez y García-Mendoza (2001) \\
Morelos & 4893 & $145 / 114$ & Bonilla-Barbosa et al. (2000) \\
Tamaulipas & & $168 / 38$ & Martínez y Novelo (1993) \\
Cuenca alta de Lerma & 5549 & $277 / 54$ & Ramos (2000) \\
\hline
\end{tabular}

En general la flora de los humedales de Lerma es comparable y en varios casos superior a la de otros ambientes lénticos de México. Por ejemplo, considerando la vegetación reportada para los lagos de la región hidrológica de la cuenca Lerma-Chapala-Santiago, a la que también pertenece la zona de estudio, se observa que las ciénegas de Lerma poseen una flora de dimensión similar a la de Zirahuén y Cuitzeo, tanto en el número total de especies (97) como en el de acuáticas estrictas (40). Pero comparada con la flora de Pátzcuaro y Yuriria resulta ser casi el doble de rica, no obstante que ambos lagos son significativamente más grandes.

Dentro del estado de México las ciénegas de Lerma albergan un número importante de plantas acuáticas. Ramos (2000) reconoce para toda la cuenca alta del río 
Lerma 277 especies de acuáticas, subacuáticas y tolerantes, sin embargo en la extensión que ocupan las ciénegas, apenas menor a 1\% del área de toda la cuenca alta (5 $549 \mathrm{~km}^{2}$ ), es posible encontrar más de la tercera parte de la flora registrada por este autor. De las 97 entidades de acuáticas, subacuáticas y tolerantes aquí enlistadas, sólo $72 \%$ fueron incluidas en los inventarios de Ramos (2000), y si se consideran únicamente las hidrófitas estrictas y las subacuáticas, el presente estudio comparte con su lista $71 \%$. De esta forma, las colectas realizadas en esta investigación han permitido incorporar a los reportes previos de la flora de las ciénegas 19 especies más de plantas acuáticas y subacuáticas y nueve de plantas tolerantes (Apéndice 1).

La presencia de diez elementos endémicos de México y de dos amenazados (Apéndice 1) es una característica que distingue la flora de las ciénegas; este tipo de especies y en general la alta diversidad que albergan los humedales de Lerma, ha sido uno de los criterios más importantes para incluirlas como áreas naturales protegidas (Anónimo, 2002a; Arriaga-Cabrera et al., 2009) y al mismo tiempo hace necesaria y urgente la creación de programas de manejo y conservación aplicables a las demandas locales. De las plantas acuáticas encontradas en la zona, actualmente Sagittaria macrophylla (papa de agua), Nymphaea gracilis (estrella de agua) y Lemna trisulca han sido incluidas en la Norma Oficial Mexicana-059- ECOL-2001 como amenazadas o sujetas a protección especial, dada su distribución restringida en México (Lot y Zepeda, 2009). De estas sólo S. macrophylla crece ampliamente en la ciénegas de Lerma, lo que probablemente contribuye a que sus semillas y tubérculos sean uno de los alimentos más importantes de la dieta del pato mexicano (Anas diazi) (Colón-Quezada, 2009).

La transformación, destrucción y desaparición de los ecosistemas acuáticos de la región han propiciado la reducción del área de distribución natural de las acuáticas antes mencionadas y de otras como Ceratophyllum demersum, Lilaea scilloides, Myriophyllum heterophyllum, Sagittaria latifolia, Ruppia maritima, Potamogeton nodosus y Wolffiella oblonga, que si bien muchas de ellas son abundantes en otras partes de México (Lot et al., 1999), en la zona de estudio son consideradas como especies raras y potencialmente amenazadas, dado que sus poblaciones tienen una distribución muy restringida (Ramos, 2000). La alteración de los sistemas acuáticos de Lerma también ha afectado a algunas especies endémicas de fauna que actualmente se encuentran amenazadas, tal es el caso de aves como el pato mexicano (Anas diazi), la mascarita transvolcánica (Geothlypis speciosa) y la polla de agua (Coturnicops noveboracens) o de anfibios como el ajolote de Lerma (Ambystoma lermaensis) y algunos peces como el charal (Chirostoma riojai) y el pupo (Algansea barbata) (Anónimo, 2002b). 
Las comunidades vegetales de las ciénegas mantienen una diversidad importante según los valores obtenidos del índice de Shannon (Cuadro 1). Chiconahuapan puede considerase como el más diverso de los tres humedales (Cuadro 2) y en función de la composición de especies es el que aportó mayor heterogeneidad florística a la región (Cuadro 2, Fig. 4).

En cada humedal prevalecen condiciones ecológicas particulares que se reflejan en la diversidad de su vegetación. Las hidrófitas enraizadas emergentes son los elementos más ampliamente distribuidos, sin embargo en cada ciénega se agrupan de formas diferentes creando asociaciones fisonómica y florísticamente heterogéneas (Fig. 2). La zonificación de estas agrupaciones en la mayoría de los casos está relacionada con la eliminación o modificación del ambiente lacustre, particularmente con el nivel de inundación, como lo sugieren los estudios que reconocen a la profundidad del agua y sus fluctuaciones (naturales o antropogénicas) como el principal determinante de la diversidad y abundancia de las plantas acuáticas (Cronk y Fennessy, 2001)

Durante la mayor parte del año Chiconahuapan tiene un nivel de inundación superior al de Chignahuapan (Pérez-Ortiz, 2005), que junto con su relativamente bajo nivel de eutroficación, pueden explicar su mayor riqueza florística y de formas de vida, así como la presencia de hidrófitas sumergidas en pequeñas áreas (Ceratophyllum demersum, Myriophyllum heterophyllum, Ruppia maritima, Potamogeton spp. y Najas guadalupensis) que con seguridad eran más extensas, no sólo en esta ciénega sino también en las otras dos.

En contraste, la sequía que afecta una buen parte de la ciénega de Chignahuapan y la consecuente ausencia de una columna de agua durante más de cuatro meses, parece ser la causa principal de que sus comunidades vegetales sean florísticamente más pobres y reducidas, particularmente las de hidrófitas sumergidas, que incapaces de tolerar este cambio están desapareciendo localmente. Las especies con amplia tolerancia a las variaciones ambientales y a la perturbación son los componentes más comunes de las asociaciones vegetales de este humedal, como algunas ciperáceas, gramíneas y poligonáceas.

Como sucede en otros cuerpos de agua de México (Ramos y Novelo, 1993; Rojas y Novelo, 1995; Madrigal-Guridi et al., 2004), la problemática ambiental que enfrenta la zona inundada de Lerma está centrada en una fuerte influencia negativa de la actividad humana. En las áreas lacustres de Lerma existen crecientes asentamientos humanos (Velasco, 2008), que junto con la ganadería, la deforestación (Orozco-Hernández et al., 2009), la contaminación (Sedeño-Díaz y López-López, 2007) y la sobreexplotación del acuífero (desde la década de los 60’s por la demanda de agua del D.F., 
Toluca y Metepec; Esteller y Delgado, 2002), han ocasionado una fuerte disminución del nivel de agua en los humedales, su fragmentación y la pérdida de hábitats.

Actualmente las ciénegas de Lerma se encuentran en un estado eutrófico variable y aunque la contaminación y la concentración de metales pesados aparentemente aún no representan riesgos para la vida acuática (Pérez-Ortiz, 2005; Sedeño-Díaz y López-López, 2007) y la salud (Pérez-Ortiz, 2005), el futuro de estos humedales y el de su riqueza biótica puede ser desalentador si el deterioro progresa.

Es un hecho que aunque aún existe un área importante que continúa anegada y cubierta de hidrófitas, las comunidades acuáticas y algunas especies locales, están desapareciendo. En este sentido es urgente tomar medidas para la protección y recuperación de estos cuerpos lacustres. Las estrategias deben encaminarse a manejar de forma integral el sistema, enfocando la atención a mantener los niveles de inundación, mejorar la calidad del agua, controlar las malezas y realizar estudios básicos sobre su diversidad, dinámica espacial y temporal, así como de alternativas de aprovechamiento (pesquero, turístico o recreativo) que beneficien de forma directa a las poblaciones humanas locales y que opten por un modelo de desarrollo social que cuide el ambiente.

\section{AGRADECIMIENTOS}

Un agradecimiento especial a la Universidad Autónoma del Estado de México, que patrocinó esta investigación a través del proyecto 2356/2006 y al Consejo Mexiquense de la Ciencia y la Tecnología por la beca otorgada a la primera autora. Al Dr. Javier Manjarrez quien revisó el manuscrito y participó activamente en las salidas de campo. A la bióloga Itzi Bautista y al M. en C. David Colón Quezada por su apoyo en el campo y el procesamiento del material. A los revisores anónimos por sus comentarios y aportaciones para mejorar la versión final.

\section{LITERATURA CITADA}

Aguirre-León, E. 2009. Orquídeas. In: Ceballos, G., R. List, G. Garduño, R. López-Cano, M. J. Muñoscano-Quintanar, E. Collado y J. E. San Román (eds.). 2009. La diversidad biológica del Estado de México: Estudio de Estado. Gobierno del Estado de México y Comisión Nacional para el Conocimiento y Uso de la Biodiversidad. México, D.F. pp. 203-208. 
Anónimo. 2002a. Decreto por el que se declara área natural protegida la región conocida como Ciénegas de Lerma en el Estado de México. Publicado en el Diario Oficial de la Federación. 27 de noviembre de 2002. Secretaria del Medio Ambiente y Recursos Naturales. México, D.F. pp. 4-12.

Anónimo. 2002b. Norma oficial mexicana NOM-059-ECOL-2001. Protección ambientalEspecies nativas de México de flora y fauna silvestres - Categorías de riesgo y especificaciones para su inclusión, exclusión o cambio - Lista de especies en riesgo. Publicado en el Diario Oficial de la Federación. 6 marzo de 2002. Secretaría del Medio Ambiente y Recursos Naturales. México, D.F.

Anónimo. 2006. The list of the wetlands of international importance. Ramsar List. The Ramsar Convention on Wetlands. En línea: http://www.ramsar.org/pdf/sitelist.pdf. Consulta 4 de mayo de 2011.

Arizmendi, M. C. y L. Márquez. 2000. Áreas de importancia para la conservación de las aves en México. Sección Mexicana del Congreso Internacional para la Preservación de las Aves y Fondo Mexicano para la Conservación de la Naturaleza. México, D.F. $440 \mathrm{pp}$.

Arriaga-Cabrera, L., V. Aguilar y J. M. Espinoza. 2009. Regiones prioritarias y planeación para la conservación de la biodiversidad. In: Capital natural de México. Vol. II: Estado de conservación y tendencias de cambio. Comisión Nacional para el Conocimiento y Uso de la Biodiversidad. México, D.F. pp. 433-457.

Bonilla-Barbosa, J. R. y A. Novelo. 1995. Manual de identificación de plantas acuáticas del Parque Nacional Lagunas de Zempoala, México. Cuaderno 26. Instituto de Biología, Universidad Nacional Autónoma de México. México, D.F. 168 pp.

Bonilla-Barbosa, J. R., J. A. Viana-Lases y F. Salazar-Villegas. 2000. Listados florísticos de México. XX. Flora acuática de Morelos. Instituto de Biología, Universidad Nacional Autónoma de México. México, D.F. 29 pp.

Brower, J. E., J. H. Zar y C. N. Von Ende. 1997. Field and laboratory methods for general ecology. 4a ed. McGraw-Hill. Quebec. 273 pp.

Brummitt, R. K. (comp.). 1992. Vascular plant families and genera. Royal Botanic Gardens. Kew. 804 pp.

Brummitt, R. K. y C. E. Powell. 1992. Authors of plant names. Royal Botanic Gardens. Kew. $732 \mathrm{pp}$.

Colón-Quezada, D. 2009. Composición de la dieta de otoño del pato mexicano (Anas diazi) en el vaso sur de las ciénegas del Lerma, estado de México. Rev. Mex. Biodiv. 80: 193-202.

Cronk, J. K. y M. S. Fennessy. 2001. Wetland plants: biology and ecology. Lewis Publishers. Boca Raton, Florida. 462 pp.

Cronquist, A. 1981. An integrated system of classification of flowering plants. Columbia University Press. New York. 1262 pp.

Dávila, P. 2009. Gramíneas. In: Ceballos, G., R. List, G. Garduño, R. López-Cano, M. J. Muñoscano-Quintanar, E. Collado y J. E. San Román. (eds.). 2009. La diversidad biológica del Estado de México: Estudio de estado. Gobierno del Estado de México y Comisión Nacional para el Conocimiento y Uso de la Biodiversidad. México, D.F. pp. 209-212. 
Espinosa, G. J. y J. Sarukhán. 1997. Manual de malezas del Valle de México. Ediciones Científicas Universitarias. Universidad Nacional Autónoma de México. México, D.F. $407 \mathrm{pp}$.

Esteller, M. V. y C. Delgado. 2002. Las aguas subterráneas en una cuenca de altura: el Valle de Toluca. Capítulo 6. In: Antón, D. y C. Díaz. Sequía en un mundo de agua. Programa Iberoamericano de Ciencia y Tecnología para el Desarrollo, Red Iberoamericana de Potabilización y Depuración del Agua, Consejo Nacional de Ciencia y Tecnología, Universidad Autónoma del Estado de México, Centro Interamericano de Recursos del Agua y Coordinación General de Investigación y Estudios Avanzados. Edición digital http://tierra.rediris.es/hidrored/ebooks/sequia/cap06.html Consulta: 01 de marzo de 2010.

Hammer, Ø., D. A. T. Harper y P. D. Ryan. 2001. PAST: paleontological statistics software package for education and data analysis. Paleon. Elec. 4(1): 9. http://paleo-electronica. org/2001_1/past/issue1_01.htm

Holland, M., F. Dennis y G. Brij. 1990. The characteristics of wetland ecotones. In: Naiman, R. I. y H. Decamps (eds.). 1990. The ecology and management of aquatic and terrestrial ecotones. MAB Series No. 4. United Nations Educational, Scientific and Cultural Organization. París. pp. 717-198.

Lot, A. y A. Novelo. 1978. Laguna de Tecocomulco, Hidalgo. Guía Botánica de Excursiones en México. Publicación Especial de la Sociedad Botánica de México, A.C. México, D.F. 19 pp.

Lot, A. y A. Novelo. 1988. Vegetación y flora acuática del Lago de Pátzcuaro, Michoacán, México. Southwest. Nat. 33(2): 167-175.

Lot, A. y A. Novelo. 2004. Iconografía y estudio de plantas acuáticas de la ciudad de México y sus alrededores. Instituto de Biología, Universidad Nacional Autónoma de México. México, D.F. 206 pp.

Lot, A., A. Novelo y P. Ramírez-García. 1986. Angiospermas acuáticas mexicanas 1. Listados florísticos de México. Instituto de Biología, Universidad Nacional Autónoma de México. México, D.F. 60 pp.

Lot, A., A. Novelo y P. Ramírez-García. 1998. Diversidad de la flora acuática mexicana. In: Ramamoorthy, T. P., R. Bye, A. Lot y J. Fa (eds.). 1998. Diversidad biológica de México. Instituto de Biología. Universidad Nacional Autónoma de México. México, D.F. pp. 563-577.

Lot, A., A. Novelo, M. Olvera y P. Ramírez-García. 1999. Catálogo de angiospermas acuáticas de México. Serie Cuadernos Núm. 33. Instituto de Biología. Universidad Nacional Autónoma de México. México, D.F. 161 pp.

Lot, A. y C. Zepeda. 2009. Plantas acuáticas. In: Ceballos, G., R. List, G. Garduño, R. López-Cano, M. J. Muñoscano-Quintanar, E. Colladoy J. E. San Román. (eds.). 2009. La diversidad biológica del Estado de México: Estudio de Estado. Gobierno del Estado de México y Nacional para el Conocimiento y Uso de la Biodiversidad. México, D.F. pp. 229-241.

Madrigal-Guridi, X., A. Novelo y A. Chacón. 2004. Flora y vegetación acuática del lago de Zirahuén, Michoacán, México. Acta Bot. Mex. 68: 1-38. 
Martínez C., Y. I. 1993. La evolución de la superficie de las lagunas de Lerma y sus principales repercusiones físicas, biológicas y humanas. Tesis de Licenciatura. Facultad de Geografía, Universidad Autónoma del Estado de México. Toluca. 119 pp.

Martínez, M. y A. García-Mendoza. 2001. Flora y vegetación acuática de localidades selectas del estado de Querétaro. Acta Bot. Mex. 54: 1-23.

Martínez, M. y A. Novelo. 1993. La vegetación acuática del estado de Tamaulipas, México. An. Inst. Biol. 64(2): 59-86.

Matteucci, S. y A. Colma. 1982. Metodología para el estudio de la vegetación. Serie de Biología, Monografía No 22. Secretaría General de la Organización de los Estados Americanos. Washington, D.C. 168 pp.

Mitsch, W. J. y J. G. Gosselink. 1993. Wetlands. Van Nostrand Reinhold. New York. 722 pp.

Mora-Olivo, A., J. L. Villaseñor, I. Luna-Vega y J. J. Morrone. 2008. Patrones de distribución de la flora vascular acuática estricta en el estado de Tamaulipas, México. Rev. Mex. Biodiv. 79: 435-448.

Moreno, C. E. 2001. Métodos para medir la biodiversidad. Manuales y tesis, Sociedad Entomológica Aragonesa. Vol. 1. Zaragoza, España. 80 pp.

Novelo, A. y M. Gallegos. 1988. Estudio de la flora y la vegetación acuática relacionada con el sistema de chinampas en el sureste del Valle de México. Biótica 13(1,2): 121-139.

Orozco-Hernández, M., G. Gutiérrez-Martínez y J. Delgado-Campos. 2009. Desarrollo rural y deterioro del bosque. Región interestatal del alto Lerma. Econ. Soc. Terr. IX(30): 435-472.

Pérez-Ortiz, G. 2005. Diagnóstico ambiental como base para la rehabilitación de las Ciénegas de Lerma, Estado de México. Tesis de maestría en Ciencias Biológicas. Universidad Nacional Autónoma de México. México, D.F. 85 pp.

Pérez-Ortiz, G. y M. Valdez. 2006. El uso de la biodiversidad en las ciénegas del Lerma. In: Cotler, A. H., M. Mazari y J. de A. Sánchez (eds.). 2006. Atlas de la Cuenca Lerma Chapala. Instituto Nacional de Ecología y Secretaria del Medio Ambiente y Recursos Naturales. México, D.F. p. 78.

Ramírez C., D. y T. Herrera. 1954. Contribución al conocimiento de la vegetación del Lerma y sus alrededores. An. Inst. Biol. 25: 65-95.

Ramos, V. L. 2000. Estudio de la flora de la vegetación acuáticas vasculares de la cuenca alta del Río Lerma, en el Estado de México. Tesis de maestría en Ecología y Ciencias Ambientales. Universidad Nacional Autónoma de México. México, D.F. 146 pp.

Ramos, V. L. y A. Novelo. 1993. Vegetación y flora acuática de la laguna de Yuriria, Guanajuato, México. Acta Bot. Mex. 25: 61-79.

Rioja, E. y T. Herrera. 1951. Ensayo ecológico del limnobio de Lerma y sus alrededores. An. Inst. Biol. 22(2): 365-351.

Rojas, M. J. y A. Novelo. 1995. Flora y vegetación acuática del lago de Cuitzeo. Michoacán, México. Acta Bot. Mex. 31: 1-17.

Rzedowski, G. C. de y J. Rzedowski. 2001. Flora fanerogámica del Valle de México. 2a. ed. Instituto de Ecología, A.C. y Comisión Nacional para el Conocimiento y Uso de la Biodiversidad. Pátzcuaro, Mich. 1406 pp. 
Rzedowski, J. 1957. Algunas asociaciones vegetales de los terrenos del lago de Texcoco. Bol. Soc. Bot. Méx. 8: 59-129.

Rzedowski, J. 1998. Diversidad y orígenes de la flora fanerogámica de México. In: Ramamoorthy, T. P., R. Bye, A. Lot y J. Fa. 1998. Diversidad biológica de México: orígenes y distribución. Instituto de Biología. Universidad Nacional Autónoma de México. México, D.F. pp. 129-145.

Sculthorpe, C. D. 1985. The biology of aquatic vascular plants. Edward Arnold, Ltd. London, England. 610 pp.

Sedeño-Díaz, J. E. y E. López-López. 2007. Water quality in the Río Lerma, Mexico: an overview of the last quarter of the twentieth century. Wat. Res. Man. 21: 1797-812.

Tejero-Díez, J. D. 2009. Helechos y licopodios. In: Ceballos, G., R. List, G. Garduño, R. López-Cano, M. J. Muñoscano-Quintanar, E. Collado y J. E. San Román. (eds.). 2009. La diversidad biológica del Estado de México: Estudio de Estado. Gobierno del Estado de México y Comisión Nacional para el Conocimiento y Uso de la Biodiversidad. México. D.F. pp. 167-176.

Tejero-Díez, J. D. y M. L. Arreguín-Sánchez. 2004. Lista con anotaciones de los pteridofitos del estado de México. Acta Bot. Mex. 69: 1-82.

Tiner, R. W. 1999. Wetlands indicators. A guide to wetland identification, delineation, classification and mapping. Lewis Publishers. New York. 392 pp.

Tryon, R. M. y A. F. Tryon. 1982. Ferns and allied plants, with special reference to tropical America. Springer Verlag. New York. 835 pp.

Vásquez, J. 1999. Almoloya del Río. Monografía municipal. Gobierno del Estado de México. Toluca. 184 pp.

Velasco, J. J. 2008. La ciénega de Chiconahuapan, Estado de México: Un humedal en deterioro constante. Contribuciones desde Coatepec 15: 101-125.

Zar, J. 1999. Biostatistical analysis. Prentice Hall. Upper Saddle River, New Jersey. 662 pp. 


\section{APÉNDICE 1}

Lista florística de la vegetación de las ciénegas de Chignahuapan, Chimaliapan y Chiconahuapan, Estado de México. Categoría: $\mathrm{A}=$ planta acuática, $\mathrm{S}=$ subacuática, $\mathrm{T}=$ tolerante, $\mathrm{R}=$ riparia. Forma de vida: $\mathrm{HEE}=$ hidrófita enraizada emergente, $\mathrm{HEHF}=$ hidrófita enraizada de hojas flotantes, HES = hidrófita enraizada sumergida, HETP = hidrófita enraizada de tallos postrados, HLS = hidrófita libre sumergida, HLF = hidrófita libre flotadora, $\mathrm{M}=$ maleza. ${ }^{\mathrm{a}}=$ familias de plantas estrictamente acuáticas, $*$ = especie endémica de México, = especie endémica de Megaméxico, $\boldsymbol{\Delta}=$ especie protegida, $\boldsymbol{\nabla}=$ amenazada, = No reportada para las ciénegas de Lerma en Rioja y Herrera (1951), Ramírez y Herrera (1954) y Ramos (2000).

\begin{tabular}{lll}
\hline Especie & Categoría & Forma de vida \\
\hline
\end{tabular}

Marsileaceae

Marsilea mollis B. L. Rob. \& Fernald

Salviniaceae

Azolla mexicana C. Presl

\section{MAGNOLIOPHYTA \\ LILIOPSIDA}

\section{Alismataceae $^{\mathrm{a}}$}

Sagittaria latifolia Willd.

$\nabla *$ Sagittaria macrophylla Zucc.

A

A

\section{Commelinaceae}

Commelina tuberosa $\mathrm{L}$.

Tinantia erecta (Jacq.) Schltdl.

$\checkmark$ Tripogandra purpurascens (S. Schauer) Handlos

\section{Cyperaceae}

Cyperus niger Ruiz \& Pavón

-*Cyperus semiochraceus Boeck.

Cyperus spectabilis Link

Eleocharis bonariensis Nees

Eleocharis densa Benth.

Eleocharis dombeyana Kunth

Eleocharis macrostachya Britton

-Eleocharis montana (Kunth) Roem. \& Schult.

Schoenoplectus americanus (Pers.) Volkart ex Schinz

\& Keller

Schoenoplectus californicus (C. A. Meyer) Soják
A

A

HEHF

HLF 
Apéndice. Continuación

\begin{tabular}{|c|c|c|}
\hline Especie & Categoría & Forma de vida \\
\hline $\begin{array}{l}\text { Schoenoplectus tabernaemontani (C. C. Gmel.) Palla } \\
\text { Hydrocharitaceae }^{\mathrm{a}}\end{array}$ & A & HEE \\
\hline $\begin{array}{l}\text { Hydromystria laevigata (Willd.) Hunz. } \\
\text { Iridaceae }\end{array}$ & A & HLF \\
\hline Sisyrinchium convolutum Nocca & $\mathrm{T}$ & \\
\hline $\begin{array}{l}\text { *Sisyrinchium tolucense Peyr. } \\
\text { Juncaceae }\end{array}$ & $\mathrm{T}$ & M \\
\hline Juncus ebracteatus Liebm. & S & HEE \\
\hline $\begin{array}{l}\text { Juncus effusus L. } \\
\text { Juncaginaceae }^{\mathbf{a}}\end{array}$ & $\mathrm{S}$ & HEE \\
\hline $\begin{array}{l}\text { Lilaea scilloides (Poir.) Hauman } \\
\text { Lemnaceae }^{\mathbf{a}}\end{array}$ & A & HEE \\
\hline Lemna gibba $\mathrm{L}$. & A & HLF \\
\hline Lemna minuscula Herter & A & HLF \\
\hline Lemna obscura (Austin) Daubs & A & HLF \\
\hline A Lemna trisulca L. & A & HLF \\
\hline Spirodela polyrrhiza (L.) Schleid. & A & HLF \\
\hline Wolffia columbiana G. Karst. & A & HLF \\
\hline Wolffiella lingulata (Hegelm.) Hegelm. & A & HLF \\
\hline $\begin{array}{l}\text { Wolffiella oblonga (Phil.) Hegelm. } \\
\text { Najadaceae }^{\mathrm{a}}\end{array}$ & A & HLF \\
\hline $\begin{array}{l}\text { Najas guadalupensis (Spreng.) Magnus } \\
\text { Poaceae }\end{array}$ & A & HES \\
\hline Echinochloa crus-pavonis (Kunth) Schult. & $\mathrm{S}$ & HEE \\
\hline Echinochloa holciformis (Kunth) Chase & $\mathrm{S}$ & HEE \\
\hline •lyceria fluitans (L.) R. Br. & S & HEE \\
\hline • Glyceria mexicana (Kelso) Beetle & $\mathrm{S}$ & HEE \\
\hline Leersia hexandra Sw. & A & HEE \\
\hline$\diamond$ Paspalum distichum L. & $\mathrm{T}$ & \\
\hline •*ennisetum crinitum (Kunth) Spreng. & $\mathrm{T}$ & \\
\hline Роа аппиа L. & $\mathrm{T}$ & M \\
\hline Pontederiaceae $^{a}$ & & \\
\hline Eichhornia crassipes (Mart.) Solms & A & HLF \\
\hline Potamogetonaceae $^{\mathrm{a}}$ & & \\
\hline Potamogeton illinoensis Morong & A & HES \\
\hline Potamogeton nodosus Poir. & A & HEHF \\
\hline Ruppiaceae $^{\mathrm{a}}$ & & \\
\hline$\checkmark$ Ruppia maritima L. & A & HES \\
\hline
\end{tabular}


Apéndice. Continuación

\begin{tabular}{|c|c|c|}
\hline Especie & Categoría & Forma de vida \\
\hline \multicolumn{3}{|l|}{ Typhaceae $^{\mathrm{a}}$} \\
\hline Typha latifolia $\mathrm{L}$. & A & HEE \\
\hline \multicolumn{3}{|l|}{ MAGNOLIOPSIDA } \\
\hline Amaranthaceae & & \\
\hline $\begin{array}{l}\text { Amaranthus hybridus L. } \\
\text { Apiaceae }\end{array}$ & $\mathrm{T}$ & M \\
\hline • Conium maculatum L. & $\mathrm{T}$ & M \\
\hline Berula erecta (Huds.) Coville & A & HEE \\
\hline Hydrocotyle aff. umbellata L. & A & HEE \\
\hline Hydrocotyle ranunculoides L. & A & HEE \\
\hline Hydrocotyle verticillata Thunb. & A & HEE \\
\hline $\begin{array}{l}\text { Lilaeopsis schaffneriana (Schltdl.) Coult. \& Rose } \\
\text { Asteraceae }\end{array}$ & A & HEE \\
\hline Aster subulatus Michx. & $\mathrm{T}$ & M \\
\hline Bidens aurea (Aiton) Sherff & $\mathrm{S}$ & HEE \\
\hline Bidens laevis (L.) Britton, Sterns \& Poggenb. & S & HEE \\
\hline Cotula australis (Sieber ex Spreng.) Hook. f. & $\mathrm{T}$ & M \\
\hline •*Euphrosyne partheniifolia DC. & S & HEE \\
\hline Gnaphalium luteo-album L. & $\mathrm{T}$ & M \\
\hline Gnaphalium semiamplexicaule DC. & $\mathrm{T}$ & M \\
\hline $\begin{array}{l}\text { *Jaegeria bellidiflora (Sessé \& Moc. ex DC.) } \\
\text { Torres \& Beaman }\end{array}$ & A & HEE \\
\hline *Jaegeria glabra (S. Watson) B.L. Rob. & A & HEE \\
\hline Melampodium bibracteatum S. Watson & S & HEE \\
\hline $\begin{array}{l}\text { \$Soliva anthemifolia (Juss.) R. Br. } \\
\text { Callitricaceae }\end{array}$ & S & HEE \\
\hline $\begin{array}{l}\text { Callitriche deflexa A. Braun ex Hegelm. } \\
\text { Caryophyllaceae }\end{array}$ & A & HEE \\
\hline Arenaria bourgaei Hemsl. & $\mathrm{S}$ & HETP \\
\hline Arenaria paludicola B. L. Rob. & S & HEE \\
\hline Cerastium brachypodum (Engelm.) B. L. Rob. & S & HEE \\
\hline Ceratophyllaceae $^{\mathrm{a}}$ & & \\
\hline Ceratophyllum demersum L. & A & HLS \\
\hline Chenopodiaceae & & \\
\hline $\begin{array}{l}\text { Chenopodium ambrosioides L. } \\
\text { Crassulaceae }\end{array}$ & $\mathrm{T}$ & M \\
\hline Tillaea saginoides Maxim. & $\mathrm{S}$ & HEE \\
\hline
\end{tabular}


Apéndice. Continuación

\begin{tabular}{|c|c|c|}
\hline Especie & Categoría & Forma de vida \\
\hline \multicolumn{3}{|l|}{ Cruciferae } \\
\hline Rorippa mexicana (Moc. \& Sessé) Standl. & $\mathrm{S}$ & HEE \\
\hline \multicolumn{2}{|l|}{ Haloragaceae } & HEE \\
\hline Myriophyllum aquaticum (Vell.) Verdc. & A & HES \\
\hline Myriophyllum heterophyllum Michx. & A & HES \\
\hline \multicolumn{3}{|l|}{ Lentibulariaceae $^{\mathrm{a}}$} \\
\hline \multicolumn{2}{|l|}{ Menyanthaceae $^{a}$} & HLS \\
\hline \multicolumn{3}{|l|}{ Nymphaeaceae $^{\mathrm{a}}$} \\
\hline 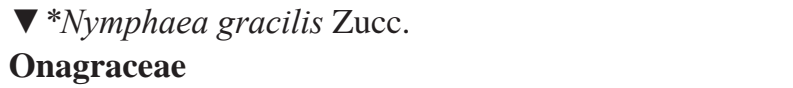 & Onagraceae & HEHF \\
\hline Epilobium ciliatum Raf. & S & HEE \\
\hline Ludwigia peploides (Kunth) P. H. Raven & A & HETP \\
\hline \multicolumn{3}{|l|}{ Plantaginaceae } \\
\hline Plantago major L. & $\mathrm{T}$ & M \\
\hline \multicolumn{3}{|l|}{ Polygonaceae } \\
\hline Polygonum hydropiperoides Michx. & S & HEE \\
\hline Polygonum lapathifolium L. & $\mathrm{S}$ & HEE \\
\hline Polygonum mexicanum Small & S & HEE \\
\hline Polygonum punctatum Elliott & S & HEE \\
\hline Rumex crispus L. & $\mathrm{T}$ & M \\
\hline \multicolumn{3}{|l|}{ Ranunculaceae } \\
\hline Ranunculus cymbalaria Pursh & S & HEE \\
\hline Ranunculus dichotomus Moc. \& Sessé & $\mathrm{T}$ & \\
\hline \multicolumn{3}{|l|}{ Salicaceae } \\
\hline \multicolumn{3}{|l|}{ Scrophulariaceae } \\
\hline Limosella aquatica L. & A & HEE \\
\hline -Mecardonia procumbens (Mill.) Small & S & HETP \\
\hline Mimulus glabratus Kunth & S & HEE \\
\hline $\begin{array}{l}\text { Veronica peregrina L. ssp. xalapensis (Kunth) Pennell } \\
\text { Solanaceae }\end{array}$ & $\mathrm{T}$ & \\
\hline *Datura ceratocaula Ortega & S & HEE \\
\hline ^Nierembergia angustifolia Kunth & $\mathrm{T}$ & \\
\hline
\end{tabular}


Apéndice. Continuación

\begin{tabular}{lcc}
\hline Especie & Categoría & Forma de vida \\
\hline Urticaceae & & \\
Urtica dioica var. angustifolia Schltdl. & $\mathrm{T}$ & $\mathrm{M}$ \\
\hline
\end{tabular}

\section{APÉNDICE 2}

Valores de importancia (VI) de las especies más abundantes en las ciénegas de Lerma.

\begin{tabular}{|c|c|c|c|c|}
\hline & \multicolumn{4}{|c|}{ VI } \\
\hline & Chignahuapan & Chimaliapan & Chiconahuapan & $\begin{array}{c}\text { Tres } \\
\text { ciénegas } \\
\text { agrupadas }\end{array}$ \\
\hline Nymphaea gracilis & & 0.1366 & & 0.0019 \\
\hline Ceratophyllum demersum & & & 0.3840 & 0.0022 \\
\hline Arenaria bourgaei & 0.2758 & & & 0.0023 \\
\hline Marsilea mollis & & & 0.3990 & 0.0023 \\
\hline Jaegeria glabra & & & 0.6229 & 0.0032 \\
\hline Rumex crispus & 0.4097 & & & 0.0033 \\
\hline Mimulus glabratus & 0.1950 & & & 0.0050 \\
\hline Bidens aurea & & & 1.2709 & 0.0065 \\
\hline $\begin{array}{l}\text { Urtica dioica } \\
\text { var. angustifolia }\end{array}$ & 0.1950 & & 1.5448 & 0.0093 \\
\hline Arenaria paludicola & 1.2206 & & & 0.0097 \\
\hline $\begin{array}{l}\text { Schoenoplectus } \\
\quad \text { tabernaemontani }\end{array}$ & & 0.3088 & 1.0220 & 0.0097 \\
\hline Cyperus semiochraceus & & & 1.8452 & 0.0103 \\
\hline Eleocharis montana & & 0.8433 & & 0.0114 \\
\hline Lilaea scilloides & 0.1797 & 0.7788 & & 0.0126 \\
\hline Rorippa pinnata & 1.6704 & & & 0.0130 \\
\hline Juncus effusus & & 0.6888 & 0.7981 & 0.0139 \\
\hline Eleocharis bonariensis & & 0.8006 & 0.7981 & 0.0155 \\
\hline Glyceria fluitans & & 1.4586 & & 0.0206 \\
\hline Polygonum hydropiperoides & & 0.2766 & 3.1161 & 0.0210 \\
\hline
\end{tabular}


Apéndice. Continuación

\begin{tabular}{|c|c|c|c|c|}
\hline & \multicolumn{4}{|c|}{ VI } \\
\hline & Chignahuapan & Chimaliapan & Chiconahuapan & $\begin{array}{c}\text { Tres } \\
\text { ciénegas } \\
\text { agrupadas }\end{array}$ \\
\hline Potamogeton nodosus & & 0.3833 & 3.0085 & 0.0214 \\
\hline Potamogeton illinoensis & & & 4.3898 & 0.0255 \\
\hline Epilobium ciliatum & 1.8408 & & 2.1441 & 0.0265 \\
\hline Nymphoides fallax & & & 5.6830 & 0.0302 \\
\hline Aster subulatus & 1.7840 & 1.3553 & & 0.0338 \\
\hline Polygonum lapathifolium & 0.6660 & 2.3544 & & 0.0338 \\
\hline Hydrocotyle verticillata & 5.3191 & & & 0.0445 \\
\hline Hydromystria laevigata & & 0.4275 & 6.6841 & 0.0448 \\
\hline Leersia hexandra & & 3.2924 & & 0.0457 \\
\hline Eichhornia crassipes & & 3.4155 & & 0.0489 \\
\hline Polygonum mexicanum & 4.9911 & 0.5835 & & 0.0489 \\
\hline Ludwigia peploides & & 0.8182 & 7.9899 & 0.0548 \\
\hline Typha latifolia & & & 13.9706 & 0.0698 \\
\hline Myriophyllum aquaticum & & 3.1111 & 5.2641 & 0.0699 \\
\hline Berula erecta & & 5.3333 & 1.5212 & 0.0853 \\
\hline Ranunculus cymbalaria & 10.6399 & & & 0.0901 \\
\hline Роа аппиа & 12.8470 & & & 0.1080 \\
\hline Eleocharis densa & 0.6856 & 5.8482 & 4.6924 & 0.1129 \\
\hline Polygonum punctatum & 3.7936 & 4.7893 & 3.1773 & 0.1144 \\
\hline Lilaeopsis schaffneriana & 7.3902 & 0.5391 & 4.1045 & 0.1215 \\
\hline $\begin{array}{l}\text { Myriophyllum } \\
\text { heterophyllum }\end{array}$ & & & 22.5294 & 0.1305 \\
\hline Echinochloa holciformis & 5.9858 & 5.8097 & & 0.1319 \\
\hline Paspalum distichum & & 7.2733 & 10.4303 & 0.1537 \\
\hline Schoenoplectus californicus & 13.4401 & & 13.6713 & 0.1939 \\
\hline Eleocharis macrostachya & 13.8239 & 6.4328 & 1.1972 & 0.2116 \\
\hline Sagittaria macrophylla & & 14.8531 & 0.3164 & 0.2159 \\
\hline Jaegeria bellidiflora & 4.8769 & 14.3617 & 5.7795 & 0.2720 \\
\hline Hydrocotyle ranunculoides & 7.7685 & 11.7248 & 9.0747 & 0.2811 \\
\hline
\end{tabular}


